\title{
联苯缩氨基胍衍生物的合成及其抗菌活性评价
}

\author{
余海红周胜超郭婷婷梁焯 \\ 陈华斌代卫凯宋明霞* \\ (井冈山大学医学部基础医学与药学学院 吉安 343009)
}

\begin{abstract}
摘要 基于查尔酮缩氨基胍衍生物的结构修饰，设计合成了一系列含联苯片段的缩氨基胍衍生物. 目标化合物的结构 通过 ${ }^{1} \mathrm{H}$ NMR、 ${ }^{13} \mathrm{C}$ NMR 和 HRMS 进行了确证, 并评价了其抗菌活性. 结果显示目标化合物对所选菌种显示出了较好 的抑制活性，最低抑菌浓度值(MIC)大都在 $0.5 \sim 8 \mu \mathrm{g} / \mathrm{mL}$. 其中, 2-((4'-溴[1,1'-二苯]-4-基)亚甲基)肼-1-甲脒(3j)的抗菌活 性最好，对所选菌株包括耐药菌均显示出强的抑菌活性，其中对金葡菌 CMCC(B) 26003、粪肠球菌 CMCC 29212 和多 药耐药金葡菌 ATCC 33591 尤为敏感，最低抑菌浓度值达到 $0.5 \mu \mathrm{g} / \mathrm{mL}$. 此外，化合物 $3 \mathrm{j}$ 表现低的细胞毒性，对正常人 体细胞 HEK $293 \mathrm{~T}$ 的 $\mathrm{IC}_{50}$ 值为 $60.90 \mu \mathrm{mol} / \mathrm{L}$. 该结果表明化合物 $\mathbf{3 j}$ 具有较好的选择性，在抗菌药物研究领域具有研究 价值.
\end{abstract}

关键词＼cjkstart缩氨基胍；联苯；抗菌活性

\section{Synthesis and Antimicrobial Activity Evaluation of Aminoguanidine Derivatives Containing a Biphenyl Moiety}

\author{
Yu, Haihong Zhou, Shengchao Guo, Tingting Liang, Zhuo \\ Chen, Huabin Dai, Weikai Song, Mingxia* \\ (Basic Medical and Pharmacy College, School of Medicine, Jinggangshan University, Ji'an 343009)
}

\begin{abstract}
A series of aminoguanidine derivatives containing a biphenyl moiety were designed, synthesized, and characterized by spectra methods using chalcone-aminoguanidine derivative as the lead compound. The antibacterial activity of the target compounds was evaluated. The results indicated that most of the target compounds showed potent inhibitory activity with the minimum inhibitory concentration values (MICs) in range of $0.5 \sim 8 \mu \mathrm{g} / \mathrm{mL}$. Among of which, 2-((4'-bromo-[1,1'biphenyl]-4-yl)methylene)hydrazine-1-carboximidamide (3j) exhibited broad-spectrum antibacterial activity, effective to all the chosen strains including two multidrug-resistant gram-positive strains, showed the most potent inhibitory against $S$. aureus CMCC(B) 26003, E. faecalis CMCC 29212 and multidrug-resistant S. aureus ATCC 33591 with a MIC value of $0.5 \mu \mathrm{g} / \mathrm{mL}$. Moreover, low cytotoxicity of compound 3j (HEK 293T, $\mathrm{IC}_{50}=60.90 \mu \mathrm{mol} / \mathrm{L}$ ) was found. These results suggested that the Compound $\mathbf{3 j}$, with high safety, was potential and valuable in the research of novel antibacterial drugs.
\end{abstract}

Keywords aminoguanidine; biphenyl; antimicrobial activity

With the increase of bacteria resistance, various drugresistant bacteria are constantly being discovered. Methicillin-resistant Staphylococcus aureus (MRSA), vancomycin-resistant enterococci (VRE), multi-drug resistant Escherichia coli, and multi-drug resistant Pseudomonas aeruginosa, causing lethal diseases worldwide and great difficulties in the treatment of community-acquired and nosocomial infections, ${ }^{[1 \sim 4]}$ severely threated global public health and result in high economic costs. ${ }^{[5]}$ A possible solution for this fact is to research and develop novel antibiotics with new structure, target and mechanism of action for the unmet needs to control the infections caused by resistant bacteria, which always is the core of attention for medicinal chemists. ${ }^{[6]}$

Biphenyls are ubiquitous structure in natural products, such as terpenes, lignans, flavonoids, petides and alka-

\footnotetext{
* Corresponding author. E-mail: freexiaoxiao83@aliyun.com

Received November 6, 2018; revised December 18, 2018; published online January 9, 2019.

Project supported by the National Natural Science Foundation of China (No. 81560561), and the Doctoral Foundation of Jinggangshan University (No. JZB1317).

国家自然科学基金(No. 81560561)和井冈山大学博士启动基金(No. JZB1317)资助项目.
} 
loids ${ }^{[7 \sim 9]}$ (Figure 1). These compounds exhibit broad-spectrum biological activities, such as significantly reducing serum glutamic pyruvic transaminase, alleviating liver pathological damage, enhancing liver detoxification, and inhibiting human immunovirus (HIV) and tumor cells. ${ }^{[10,11]}$ Biphenyls have attracted more and more attention in drugs, design and research because of their strong physiological activities. Many drugs with biphenyl structure have been developed, such as non-steroidal anti-inflammatory drugs felbinac, flurbiprofen, fenbufen, antipyretic analgesics diflunisal, and antibacterial drugs bifonazole ${ }^{[12]}$ (Figure 1).

Aminoguanidine derivatives, a kind of good metal anti-cancer and antiviral coordination complexes with low toxicity, have recently been investigated as antimicrobial agents. ${ }^{[13-18]}$ Wei et al. ${ }^{[13]}$ reported that a series of chalcone derivatives containing an aminoguanidine moiety (Compound I, Figure 2) showed potent antibacterial and antifungal activity against four gram-positive strains, four gram-negative strains and four clinical isolates of multidrug-resistant gram-positive bacterial strains with MICs in range of $1 \sim 16 \mu \mathrm{g} / \mathrm{mL}$. To obtain more effective antibacterial agents with novel skeleton, in this study, the fragment-based design was reported using compound $\mathbf{I}$ as the lead compound, in which the modification was focused on changing the chalcone moiety to a biphenyl group and simultaneously introducing some $\mathrm{F}, \mathrm{Cl}$ and $\mathrm{Br}$ substituents into the terminal phenyl ring. In this design, the two active fragments were hybridized into one molecule to expect a better activity. Thus, a series of new aminoguanidine derivatives containing a biphenyl moiety were synthesized, characterized and screened for their anti-bacterial and cytotoxicity.

\section{Results and discussion}

\subsection{Chemistry}

The synthetic route to prepare a new class of aminoguanidine-linked biphenyl derivatives from (4-formylphenyl) boronic acid and substituted bromobenzene is depicted in Scheme 1. The reaction of (4-formylphenyl)boronic acid with substituted bromobenzene in the presence of potassium phosphate and palladium(II) acetate in $\mathrm{N}, \mathrm{N}$ dimethylformamide-water condition produced 4'-substituent-[1,1'-biphenyl]-4-carbaldehydes $(\mathbf{2} \mathbf{a} \sim \mathbf{2 j})$ under the protection of nitrogen. Then target compounds $\mathbf{3} \mathbf{a} \sim \mathbf{3} \mathbf{j}$ were prepared by the condensation of $\mathbf{2} \mathbf{a} \sim \mathbf{2} \mathbf{j}$ with aminoguanidines. Finally, the structures of the target compounds were well characterized by ${ }^{1} \mathrm{H} \mathrm{NMR},{ }^{13} \mathrm{C} \mathrm{NMR}$, and high-resolution mass spectrometry.<smiles>COc1c2c(cc3c1-c1c(cc4c(c1OC)OCO4)CC(C)C(C)C3)OCO2</smiles>

Schizandrin C<smiles>COC(=O)c1cc(OC)c2c(c1-c1c(C(=O)OC)cc(OC)c3c1OCO3)OCO2</smiles>

Bifendate<smiles>COc1cc(C)cc2c(-c3c(O)cc(O)c4c3C[C@H](C)N[C@H]4C)ccc(O)c12</smiles>

korupensamine $\mathrm{A}$<smiles>COc1cc2c(c(OC)c1OC)-c1cc3c(cc1C[C@H]1COC(=O)[C@H]1C2)OCO3</smiles>

Steganone<smiles>c1ccc(-c2ccc(C(c3ccccc3)n3ccnc3)cc2)cc1</smiles>

Bifonazole

Felbinac<smiles>CC(C(=O)O)c1ccc(-c2ccccc2)c(F)c1</smiles>

Flurbiprofen<smiles>O=C(O)c1cc(-c2ccc(F)cc2F)ccc1O</smiles>

Diflunisal<smiles>O=C(O)CCC(=O)c1ccc(-c2ccccc2)cc1</smiles>

Fenbufen

Figure 1 Structures of classical natural products and pharmaceuticals containing biphenyl fragment<smiles>[R]c1ccc(-c2ccc(/C=N/NC(=N)N)cc2)cc1</smiles>

Figure 2 Lead compound and structure-based design of the target compounds 


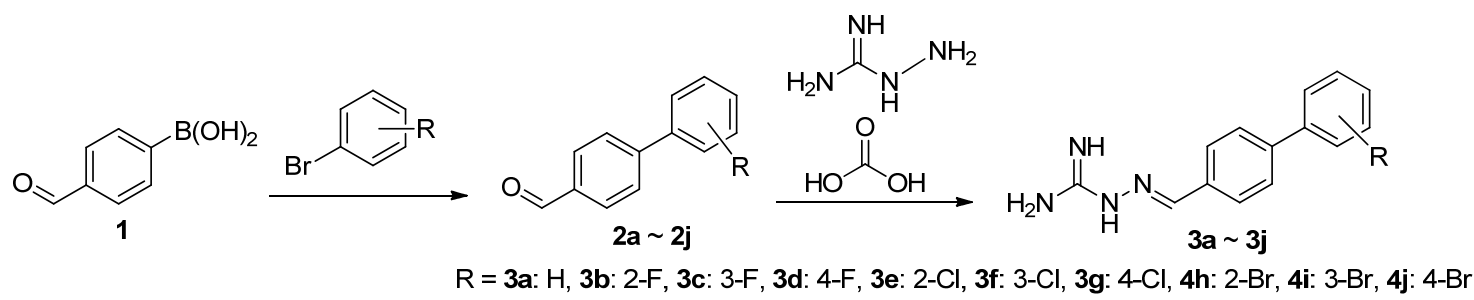

Scheme 1 Synthetic route of aminoguanidine-linked biphenyl derivatives

Taking compound 3a as an example in the structure confirmation. In the ${ }^{1} \mathrm{H}$ NMR spectrum, two singlets due to $\mathrm{N}-\mathrm{H}$ of guanidyl were observed at $\delta 5.61$ and 6.01 , which were assigned to two $\mathrm{NH}_{2}$ groups in terminal formed by the tautomerism of guanidyl group leading to the extension of conjugated chain. And the aromatic protons of terminal benzene ring were observed in $\delta 7.34 \sim 7.70$. Two doublets $(J=8.4 \mathrm{~Hz})$ due to aromatic protons of para-substituted phenyl ring were observed at $\delta 7.64$ and 7.76. The absorption peak of $\mathrm{C}-\mathrm{H}$ in imine was found at $\delta 8.04$. The absorption peak in the hydrogen spectrum is completely in conformity with the hydrogen signal in the structure. ${ }^{13} \mathrm{C}$ NMR spectra also give accurate information about the structure of the compound, which involved 10 kinds of carbon in different chemical environments. Moreover, the high-resolution mass spectrometry of $\mathbf{3 a}$ displayed an $[\mathrm{M}+\mathrm{H}]^{+}$signal at $m / z 239.1291$, which was corresponding to its molecular weight of 239.1295.

\subsection{Antimicrobial activity}

All of the target compounds $\mathbf{3} \mathbf{a} \sim \mathbf{3} \mathbf{j}$ were evaluated for their in vitro anti-bacterial activity using a serial dilution method to obtain the minimum inhibitory concentration (MIC) against five gram-positive strains (Staphylococcus aureus CMCC(B) 26003 and CMCC 25923, Streptococcus mutans BNCC 336931, Enterococcus faecalis CMCC 29212 and Bacillus subtilis CMCC 63501), and four gram-negative strains (Escherichia coli CMCC 25922 and CMCC 44568, and Pseudomonas aeruginosa CMCC 27853 and CMCC 10104) as well as two methicillin-resistant clinical isolates (Staphylococcus aureus ATCC 43300 and ATCC 33591). Gatifloxacin, moxifloxacin, norfloxacin, oxacillin, and penicillin were used as positive control drugs.

The results of target compounds $\mathbf{3 a} \sim \mathbf{3} \mathbf{j}$ were described in Tables 1, 2 as MIC values against the Gram-positive and Gram-negative strains. It could be found that most of the tested compounds showed potent inhibitory effects against the strains with MICs in $0.5 \sim 8 \mu \mathrm{g} / \mathrm{mL}$, only compounds 3a, 3f, and $\mathbf{3 h}$ showed inhibitory activity at $128 \mu \mathrm{g} / \mathrm{mL}$ against $S$. aureus CMCC(B) 26003. Compound 3j showed the most potent inhibitory activity against $S$. aureus CMCC(B) 26003 and E. faecalis CMCC 29212 with a MIC value of $0.5 \mu \mathrm{g} / \mathrm{mL}$, which was chosen to evaluate the antibacterial activity against two multidrug-resistant Gram-positive strains ( $S$. aureus ATCC 43300 and ATCC 33591). As shown in Table 3, compound $\mathbf{3 j}$ also presented high activities (MIC $=4$ or $0.5 \mu \mathrm{g} / \mathrm{mL}$, respectively) against
Table 1 Inhibitory activity (MIC, $\left.\mu \mathrm{g} \cdot \mathrm{mL}^{-} 1\right)$ of compounds $\mathbf{3 a} \sim \mathbf{3 j}$ against Gram-positive bacteria

\begin{tabular}{|c|c|c|c|c|c|c|}
\hline \multirow{2}{*}{ Compd. } & \multirow{2}{*}{$\mathrm{R}$} & \multicolumn{5}{|c|}{ Gram-positive strains } \\
\hline & & $26003^{a}$ & $25923^{b}$ & $336931^{c}$ & $29212^{d}$ & $63501^{e}$ \\
\hline $3 \mathbf{a}$ & $\mathrm{H}$ & 128 & 4 & 16 & 8 & 8 \\
\hline $\mathbf{3 b}$ & $2-\mathrm{F}$ & 8 & 8 & 8 & 4 & 8 \\
\hline $3 \mathbf{c}$ & $3-\mathrm{F}$ & 4 & 8 & 8 & 4 & 4 \\
\hline 3d & $4-\mathrm{F}$ & 4 & 8 & 8 & 4 & 8 \\
\hline $3 \mathbf{e}$ & $2-\mathrm{Cl}$ & 2 & 8 & 8 & 4 & 4 \\
\hline 3f & $3-\mathrm{Cl}$ & 128 & 8 & 4 & 2 & 2 \\
\hline $3 g$ & $4-\mathrm{Cl}$ & 1 & 4 & 2 & 1 & 2 \\
\hline $3 \mathbf{h}$ & $2-\mathrm{Br}$ & 128 & 4 & 8 & 4 & 4 \\
\hline $3 \mathbf{i}$ & $3-\mathrm{Br}$ & 2 & 4 & 4 & 2 & 2 \\
\hline $3 \mathbf{j}$ & $4-\mathrm{Br}$ & 0.5 & 4 & 1 & 0.5 & 1 \\
\hline Gatifloxacin & - & 0.125 & 0.125 & 1 & 1 & 2 \\
\hline Moxifloxacin & - & 0.125 & 0.125 & 0.5 & 1 & 2 \\
\hline Norfloxacin & - & 0.125 & 0.125 & 16 & 1 & 2 \\
\hline Oxacillin & - & 0.125 & 0.125 & 0.125 & 128 & $>128$ \\
\hline Penicillin & - & 0.125 & 0.125 & 0.125 & 128 & 128 \\
\hline
\end{tabular}
29212; ${ }^{e}$ Bacillus subtilis CMCC 63501.

Table 2 Inhibitory activity (MIC, $\mu \mathrm{g} \bullet \mathrm{mL}^{-1}$ ) of compounds $\mathbf{3 a} \sim \mathbf{3 j}$ against Gram-negative bacteria

\begin{tabular}{llrrrr}
\hline \multirow{2}{*}{ Compd. } & \multirow{2}{*}{$\mathrm{R}$} & \multicolumn{4}{c}{ Gram-negative strains } \\
\cline { 3 - 6 } & & $25922^{a}$ & $44568^{b}$ & $27853^{c}$ & $10104^{d}$ \\
\hline 3a & $\mathrm{H}$ & 16 & 16 & 16 & 16 \\
3b & $2-\mathrm{F}$ & 8 & 8 & 16 & 8 \\
3c & $3-\mathrm{F}$ & 8 & 8 & 16 & 16 \\
3d & $4-\mathrm{F}$ & 16 & 16 & 16 & 16 \\
3e & $2-\mathrm{Cl}$ & 8 & 8 & 16 & 8 \\
3f & $3-\mathrm{Cl}$ & 4 & 8 & 8 & 8 \\
3g & $4-\mathrm{Cl}$ & 4 & 8 & 8 & 4 \\
3h & $2-\mathrm{Br}$ & 8 & 8 & 16 & 8 \\
3i & $3-\mathrm{Br}$ & 4 & 4 & 16 & 4 \\
3j & $4-\mathrm{Br}$ & 2 & 4 & 16 & 2 \\
Gatifloxacin & - & 0.125 & 0.125 & 2 & 2 \\
Moxifloxacin & - & 0.125 & 0.125 & 2 & 4 \\
Norfloxacin & - & 0.125 & 0.125 & 2 & 4 \\
Oxacillin & - & 128 & $>128$ & $>128$ & 128 \\
Penicillin & - & 128 & $>128$ & $>128$ & 32 \\
\hline
\end{tabular}

${ }^{a}$ Escherichia coli CMCC $25922 ;{ }^{b}$ Escherichia coli CMCC 44568; ${ }^{c}$ Pseudomonas aeruginosa CMCC 27853; ${ }^{d}$ Pseudomonas aeruginosa CMCC 10104.

two multidrug-resistant $S$. aureus, which were slightly less active than gatifloxacin, moxifloxacin or norfloxacin $(\mathrm{MIC}=0.5$ or $0.25 \mu \mathrm{g} / \mathrm{mL}$ ) but more potent than oxacillin $(\mathrm{MIC}=64$ or $8 \mu \mathrm{g} / \mathrm{mL})$ and penicillin $(\mathrm{MIC} \geqslant 32 \mu \mathrm{g} / \mathrm{mL})$. 
Table 3 Inhibitory activity (MIC, $\mu \mathrm{g} \cdot \mathrm{mL}^{-1}$ ) of compounds $\mathbf{3 j}$ against clinical isolates of multidrug-resistant strains

\begin{tabular}{lccc}
\hline \multirow{2}{*}{ Compd. } & $\mathrm{R}$ & \multicolumn{2}{c}{$\begin{array}{c}\text { Multidrug-resistant } \\
\text { Gram-positive strains }\end{array}$} \\
\cline { 2 - 4 } & & $43300^{a}$ & $33591^{b}$ \\
\hline $\mathbf{3 j}$ & $4-\mathrm{Br}$ & 4 & 0.5 \\
Gatifloxacin & - & 0.5 & 0.25 \\
Moxifloxacin & - & 0.5 & 0.25 \\
Norfloxacin & - & 0.5 & 0.25 \\
Oxacillin & - & 64 & 8 \\
Penicillin & - & 32 & $>32$ \\
\hline${ }^{a}$ Staphylococcus aureus ATCC 43300; ${ }^{b}$ Staphylococcus aureus ATCC 33591.
\end{tabular}

In this study, some simple structure activity relationship patterns could be found between the antibacterial activity and the position and physicochemical properties of the different substituents on the phenyl ring. Generally, the halogen atom on different positions of phenyl ring played an advantageous role in increasing the antimicrobial activity relative to the non-substituted examples. A comparison of the halogen derivatives at the 4-position of the phenyl ring indicated that the different halogen atoms contributed to the antimicrobial activity in the order of $\mathrm{Br}>\mathrm{Cl}>\mathrm{F}$. The position of the $\mathrm{Br}$ substituent on the phenyl ring also affected the activity of the compounds with an activity order of $4-\mathrm{Br}>3-\mathrm{Br}>2-\mathrm{Br}$.

\subsection{Cytotoxic activity}

The cytotoxic property of compound $\mathbf{3 j}$ was also investigated using CCK-8 test against one human normal cell lines HEK 293T. Interestingly, compound 3j showed low cytotoxic activity with an $\mathrm{IC}_{50}$ value of $60.90 \mu \mathrm{mol} / \mathrm{L}$. This result indicated that this compound has non-toxicity in normal cells at effective dose, suggesting a potential for a good therapeutic index when using as anti-bacterial agents.

\section{Conclusions}

In the present work, a series of aminoguanidine derivatives containing a biphenyl moiety were synthesized, characterized, and screened for antimicrobial. Most of the tested compounds showed potent inhibitory activities against the selected strains with MICs in $0.5 \sim 8 \mu \mathrm{g} / \mathrm{mL}$. Among of which, compound $\mathbf{3 j}$ showed the most potent inhibitory with a MIC value of $0.5 \mu \mathrm{g} / \mathrm{mL}$ against $S$. aureus CMCC(B) 26003 and E. faecalis CMCC 29212. Compound 3j also exhibited potent inhibition against two multidrugresistant $S$. aureus ATCC 43300 and ATCC 33591 (MIC= 4 or $0.5 \mu \mathrm{g} / \mathrm{mL}$, respectively), which were slightly less active than gatifloxacin, moxifloxacin and norfloxacin $(\mathrm{MIC}=0.5$ or $0.25 \mu \mathrm{g} / \mathrm{mL}$ ) but much more potent than oxacillin $(\mathrm{MIC}=64$ or $8 \mu \mathrm{g} / \mathrm{mL})$ and penicillin $(\mathrm{MIC} \geqslant 32$ $\mu \mathrm{g} / \mathrm{mL})$. Furthermore, compound $\mathbf{3 j}$ also exhibited low degree of cytotoxicity. These findings strongly support the assumption that aminoguanidine-biphenyl conjugates could be used as a lead for further optimization of new antimicrobial agents to achieve promising therapeutics.

\section{Experimental}

\subsection{Instruments and reagents}

All of the reagents and solvents were purchased from Aladdin (Shanghai, China) or Sinopharm Chemical Reagent Co. Ltd. (Shanghai, China), and were used as received. Melting points were determined in open capillary tubes and are uncorrected. Reaction courses were monitored by thinlayer chromatography on silica gel-precoated $\mathrm{F}_{254}$ plates (Merck, Darmstadt, Germany). Developed plates were examined with UV lamps $(254 \mathrm{~nm})$. Nuclear magnetic resonance spectroscopy was performed on an AV-300 spectrometer (Bruker, Zurich, Switzerland) operating at 400 $\mathrm{MHz}$ for ${ }^{1} \mathrm{H}$ NMR and $100 \mathrm{MHz}$ for ${ }^{13} \mathrm{C} \mathrm{NMR}$, and using DMSO- $d_{6}$ as solvent and tetramethylsilane as the internal standard. Electrospray Ionization Mass Spectrometry (ESI-MS) experiments were performed on an IT-TOF mass spectrometer (Shimadzu, Tokyo, Japan) in negative ion mode.

\subsection{Synthesis method and spectral data}

4.2.1 Synthesis of 4'-substituent-[1,1'-biphenyl]-4carbaldehydes $(\mathbf{2} \mathbf{a} \sim \mathbf{2} \mathbf{j})$

To a stirred solution of (4-formylphenyl) boronic acid (7.99 mmol, 1.5 equiv.) in $N, N$-dimethylformamide (DMF) $(7 \mathrm{~mL})$ and water $(7 \mathrm{~mL})$ was added substituted bromobenzene (5.35 mmol, 1 equiv.), $\mathrm{K}_{3} \mathrm{PO}_{4}$ (2.26 g, $10.67 \mathrm{mmol}$, 2 equiv. $)$, and $\mathrm{Pd}(\mathrm{OAc})_{2}(60 \mathrm{mg}, 0.27 \mathrm{mmol}, 0.05$ equiv.) under $\mathrm{N}_{2}$. The resulting solution was stirred overnight at room temperature before the addition of water $(10 \mathrm{~mL})$. The mixture was extracted with ethyl acetate $(30 \mathrm{~mL} \times 2)$. The organic solution was dried over sodium sulfate, filtered and concentrated under vacuum. The crude residue was applied onto a silica gel column eluted with $1 \% \sim 2 \%$ ethyl acetate in petroleum ether to afford 4'-substituent-(1,1'- biphenyl)4-carbaldehydes $(\mathbf{2} \mathbf{a} \sim \mathbf{2} \mathbf{j})$ as an off white solid (yield $18 \% \sim 60 \%$ ). The ${ }^{1} \mathrm{H}$ NMR characteristic spectra of intermediates $\mathbf{2} \mathbf{a} \sim \mathbf{2} \mathbf{j}$ were provided in the supporting information.

4.2.2 Synthesis of 2-((4'-substituent-[1,1'-biphenyl]4-yl)methylene)hydrazine-1-carboximidamides (3a 〜 3j)

To a stirred solution of hydrazinecarboximidamide carbonate (353.88 mg, $2.6 \mathrm{mmol}, 1.3$ equiv.) in water $(5 \mathrm{~mL})$ was added $\mathrm{NaOAc}(213.2 \mathrm{mg}, 2.6 \mathrm{mmol}, 1.3$ equiv.). After stirring for $0.5 \mathrm{~h}$ at room temperature, a mixture of 4'-substituent-(1,1'-biphenyl)-4-carbaldehydes $(\mathbf{2} \mathbf{a} \sim \mathbf{2} \mathbf{j})$ (2 mmol, 1 equiv. $)$ in EtOH $(5 \mathrm{~mL})$ was added. Then the resulting solution was stirred at $70{ }^{\circ} \mathrm{C}$ for $8 \mathrm{~h}$. The reaction mixture was diluted with water $(15 \mathrm{~mL})$ and then cooled to room temperature. After stirred for $3 \mathrm{~h}$, large amount of solids was precipitated. The solids were collected by filtration, washed with $\mathrm{EtOH}(0.4 \mathrm{~mL} \times 2)$, and then dried in an oven under reduced pressure to afford 2-((4'-substituent(1,1'-biphenyl)-4-yl)methylene)hydrazine-1-carboximidamides $(\mathbf{3 a} \sim \mathbf{3 j}$ ) as a light yellow solid (yield 65\% 93\%).

2-([1,1'-Biphenyl]-4-yl)methylene)hydrazine-1-carboximidamide (3a): Light yellow solid, yield 93\%. m.p. 211 
$213{ }^{\circ} \mathrm{C} ;{ }^{1} \mathrm{H}$ NMR (DMSO- $\left.d_{6}, 400 \mathrm{MHz}\right) \delta: 5.61$ (s, 2H, $\left.\mathrm{NH}_{2}\right), 6.01\left(\mathrm{~s}, 2 \mathrm{H}, \mathrm{NH}_{2}\right), 7.34 \sim 7.70(\mathrm{~m}, 5 \mathrm{H}, \mathrm{ArH}), 7.64(\mathrm{~d}$, $J=8.4 \mathrm{~Hz}, 2 \mathrm{H}, \mathrm{ArH}), 7.76(\mathrm{~d}, J=8.4 \mathrm{~Hz}, 2 \mathrm{H}, \mathrm{ArH}), 8.04$ (s, $1 \mathrm{H}, \mathrm{N}=\mathrm{CH}$ ); ${ }^{13} \mathrm{C}$ NMR (DMSO- $\left.d_{6}, 100 \mathrm{MHz}\right) \delta: 160.60$, $142.80,139.83,139.25,136.18,128.98,127.42,126.82$, 126.62, 126.48; MS m/z: $239(\mathrm{M}+1)^{+}$; ESI-HRMS calcd for $\mathrm{C}_{14} \mathrm{H}_{15} \mathrm{~N}_{4}\left([\mathrm{M}+\mathrm{H}]^{+}\right)$239.1291, found 239.1295.

2-((2'-Fluoro-[1,1'-biphenyl]-4-yl)methylene)hydrazine-1-carboximidamide (3b): Light yellow solid, yield $86 \%$. m.p. 203 $206{ }^{\circ} \mathrm{C} ;{ }^{1} \mathrm{H}$ NMR (DMSO- $\left.d_{6}, 400 \mathrm{MHz}\right) \delta$ : $5.65\left(\mathrm{~s}, 2 \mathrm{H}, \mathrm{NH}_{2}\right), 6.02\left(\mathrm{~s}, 2 \mathrm{H}, \mathrm{NH}_{2}\right), 7.29 \sim 7.58(\mathrm{~m}, 6 \mathrm{H}$, $\operatorname{ArH}), 7.79$ (d, $J=8.3 \mathrm{~Hz}, 2 \mathrm{H}, \mathrm{ArH}), 8.06(\mathrm{~s}, 1 \mathrm{H}, \mathrm{N}=\mathrm{CH})$; ${ }^{13} \mathrm{C}$ NMR (DMSO- $\left.d_{6}, 100 \mathrm{MHz}\right) \delta: 161.21,159.59(\mathrm{~d}$, $\left.{ }^{1} J_{\mathrm{C}-\mathrm{F}}=218.6 \mathrm{~Hz}\right), 142.99,137.04,134.58,131.03(\mathrm{~d}$, $\left.{ }^{4} J_{\mathrm{C}-\mathrm{F}}=3.1 \mathrm{~Hz}\right), 129.90\left(\mathrm{~d},{ }^{3} J_{\mathrm{C}-\mathrm{F}}=8.3 \mathrm{~Hz}\right), 129.23(\mathrm{~d}$, $\left.{ }^{4} J_{\mathrm{C}-\mathrm{F}}=2.8 \mathrm{~Hz}\right), 128.47\left(\mathrm{~d},{ }^{3} J_{\mathrm{C}-\mathrm{F}}=12.9 \mathrm{~Hz}\right), 126.81,125.42$ $\left(\mathrm{d},{ }^{4} J_{\mathrm{C}-\mathrm{F}}=3.2 \mathrm{~Hz}\right), 116.61\left(\mathrm{~d},{ }^{2} J_{\mathrm{C}-\mathrm{F}}=22.5 \mathrm{~Hz}\right) ; \mathrm{MS} \mathrm{m} / \mathrm{z}$ : $257(\mathrm{M}+1)^{+}$. ESI-HRMS calcd for $\mathrm{C}_{14} \mathrm{H}_{14} \mathrm{FN}_{4}\left([\mathrm{M}+\mathrm{H}]^{+}\right)$ 257.1197, found 257.1195.

2-((3'-Fluoro-[1,1'-biphenyl]-4-yl)methylene)hydrazine1-carboximidamide (3c): Light yellow solid, yield 79\%. m.p. $210 \sim 212{ }^{\circ} \mathrm{C} ;{ }^{1} \mathrm{H}$ NMR (DMSO- $\left.d_{6}, 400 \mathrm{MHz}\right) \delta: 5.75$ $\left(\mathrm{s}, 2 \mathrm{H}, \mathrm{NH}_{2}\right), 6.10\left(\mathrm{~s}, 2 \mathrm{H}, \mathrm{NH}_{2}\right), 7.16 \sim 7.57$ (m, 6H, ArH), 7.69 (d, $J=8.4 \mathrm{~Hz}, 2 \mathrm{H}, \mathrm{ArH}), 7.79$ (d, $J=8.4 \mathrm{~Hz}, 2 \mathrm{H}, \mathrm{ArH})$, $8.05(\mathrm{~s}, 1 \mathrm{H}, \mathrm{N}=\mathrm{CH}) ;{ }^{13} \mathrm{C} \mathrm{NMR}\left(\mathrm{DMSO}-d_{6}, 100 \mathrm{MHz}\right) \delta$ : $163.22\left(\mathrm{~d},{ }^{1} J_{\mathrm{C}-\mathrm{F}}=241.7 \mathrm{~Hz}\right), 160.99,143.11,142.73(\mathrm{~d}$, $\left.{ }^{3} J_{\mathrm{C}-\mathrm{F}}=7.7 \mathrm{~Hz}\right), 138.22\left(\mathrm{~d},{ }^{4} J_{\mathrm{C}-\mathrm{F}}=1.8 \mathrm{~Hz}\right), 137.16,131.29$ $\left(\mathrm{d},{ }^{3} J_{\mathrm{C}-\mathrm{F}}=8.5 \mathrm{~Hz}\right), 127.28,127.21,122.96,114.50(\mathrm{~d}$, $\left.{ }^{2} J_{\mathrm{C}-\mathrm{F}}=21.1 \mathrm{~Hz}\right), 113.59\left(\mathrm{~d},{ }^{2} J_{\mathrm{C}-\mathrm{F}}=21.8 \mathrm{~Hz}\right) ; \mathrm{MS} \mathrm{m} / \mathrm{z}: 257$ $(\mathrm{M}+1)^{+}$. ESI-HRMS calcd for $\mathrm{C}_{14} \mathrm{H}_{14} \mathrm{FN}_{4}\left([\mathrm{M}+\mathrm{H}]^{+}\right)$ 257.1197, found 257.1191.

2-((4'-Fluoro-[1,1'-biphenyl]-4-yl)methylene)hydrazine1-carboximidamide (3d): Light yellow solid, yield $68 \%$. m.p. $216 \sim 218{ }^{\circ} \mathrm{C} ;{ }^{1} \mathrm{H}$ NMR (DMSO- $\left.d_{6}, 400 \mathrm{MHz}\right) \delta: 5.58$ (s, 2H, $\mathrm{NH}_{2}$ ), 5.99 (s, 2H, $\left.\mathrm{NH}_{2}\right), 7.27 \sim 7.77$ (m, 6H, ArH), $7.62(\mathrm{~d}, J=8.3 \mathrm{~Hz}, 2 \mathrm{H}, \mathrm{ArH}), 7.79$ (d, $J=8.3 \mathrm{~Hz}, 2 \mathrm{H}, \mathrm{ArH})$, $8.03(\mathrm{~s}, 1 \mathrm{H}, \mathrm{N}=\mathrm{CH}) ;{ }^{13} \mathrm{C}$ NMR (DMSO- $\left.d_{6}, 100 \mathrm{MHz}\right) \delta$ : $161.84\left(\mathrm{~d},{ }^{1} J_{\mathrm{C}-\mathrm{F}}=243.3 \mathrm{~Hz}\right), 160.62,142.68,138.14$, $136.31\left(\mathrm{~d},{ }^{4} J_{\mathrm{C}-\mathrm{F}}=3.0 \mathrm{~Hz}\right), 136.17,128.44\left(\mathrm{~d},{ }^{3} J_{\mathrm{C}-\mathrm{F}}=8.0\right.$ $\mathrm{Hz}), 126.79,126.55,115.73\left(\mathrm{~d},{ }^{2} J_{\mathrm{C}-\mathrm{F}}=21.0 \mathrm{~Hz}\right.$ ); MS $m / z$ : $257(\mathrm{M}+1)^{+}$. ESI-HRMS calcd for $\mathrm{C}_{14} \mathrm{H}_{14} \mathrm{FN}_{4}\left([\mathrm{M}+\mathrm{H}]^{+}\right)$ 257.1197, found 257.1201.

2-((2'-Chloro-[1,1'-biphenyl]-4-yl)methylene)hydrazine1-carboximidamide (3e): Light yellow solid, yield 73\%. m.p. $226 \sim 228{ }^{\circ} \mathrm{C} ;{ }^{1} \mathrm{H}$ NMR (DMSO- $\left.d_{6}, 400 \mathrm{MHz}\right) \delta: 5.59$ (s, $\left.2 \mathrm{H}, \mathrm{NH}_{2}\right), 5.99$ (s, 2H, $\left.\mathrm{NH}_{2}\right), 7.38 \sim 7.58$ (m, 6H, ArH), 7.69 (d, $J=8.2 \mathrm{~Hz}, 2 \mathrm{H}, \mathrm{ArH}), 7.77$ (d, $J=8.4 \mathrm{~Hz}, 2 \mathrm{H}, \mathrm{ArH})$, $8.05(\mathrm{~s}, 1 \mathrm{H}, \mathrm{N}=\mathrm{CH}) ;{ }^{13} \mathrm{C}$ NMR (DMSO- $\left.d_{6}, 100 \mathrm{MHz}\right) \delta$ : $160.74,142.53,139.58,137.76,136.49,131.41,131.25$, 129.91, 129.27, 029.09, 127.54, 125.93. MS m/z: $273(\mathrm{M}+$ $1)^{+}$. ESI-HRMS calcd for $\mathrm{C}_{14} \mathrm{H}_{14} \mathrm{ClN}_{4}\left([\mathrm{M}+\mathrm{H}]^{+}\right)$273.0902, found 273.0900 .

2-((3'-Chloro-[1,1'-biphenyl]-4-yl)methylene)hydrazine1-carboximidamide (3f): Light yellow solid, yield $65 \%$. m.p. $208 \sim 210{ }^{\circ} \mathrm{C} ;{ }^{1} \mathrm{H}$ NMR (DMSO- $\left.d_{6}, 400 \mathrm{MHz}\right) \delta: 5.69$ $\left(\mathrm{s}, 2 \mathrm{H}, \mathrm{NH}_{2}\right), 6.06\left(\mathrm{~s}, 2 \mathrm{H}, \mathrm{NH}_{2}\right), 7.39 \sim 7.79(\mathrm{~m}, 8 \mathrm{H}, \mathrm{ArH})$, $8.04(\mathrm{~s}, 1 \mathrm{H}, \mathrm{N}=\mathrm{CH}) ;{ }^{13} \mathrm{C} \mathrm{NMR}$ (DMSO- $\left.d_{6}, 100 \mathrm{MHz}\right) \delta$ :
$160.63,142.58,141.98,137.56,136.80,133.80,130.77$, $127.19,126.84,126.80,126.14,125.16$; MS m/z: $273(\mathrm{M}+$ $1)^{+}$. ESI-HRMS calcd for $\mathrm{C}_{14} \mathrm{H}_{15} \mathrm{ClN}_{4}\left([\mathrm{M}+\mathrm{H}]^{+}\right)$273.0902, found 273.0905 .

2-((4'-Chloro-[1,1'-biphenyl]-4-yl)methylene)hydrazine1-carboximidamide (3g): Light yellow solid, yield 70\%. m.p. $197 \sim 200{ }^{\circ} \mathrm{C} ;{ }^{1} \mathrm{H}$ NMR (DMSO- $d_{6}, 400 \mathrm{MHz}$ ) $\delta: 5.64$ $\left(\mathrm{s}, 2 \mathrm{H}, \mathrm{NH}_{2}\right), 6.03\left(\mathrm{~s}, 2 \mathrm{H}, \mathrm{NH}_{2}\right), 7.51(\mathrm{~d}, J=8.6 \mathrm{~Hz}, 2 \mathrm{H}$, $\operatorname{ArH}), 7.65$ (d, $J=8.4 \mathrm{~Hz}, 2 \mathrm{H}, \mathrm{ArH}), 7.66(\mathrm{~d}, J=8.6 \mathrm{~Hz}, 2 \mathrm{H}$, $\operatorname{ArH}), 7.78$ (d, J=8.40 Hz, 2H, ArH), 8.03 (s, 1H, N=CH); ${ }^{13} \mathrm{C}$ NMR (DMSO- $\left.d_{6}, 100 \mathrm{MHz}\right) \delta: 160.63,142.65,138.62$, $137.81,136.53,132.22,128.91,128.22,126.86,126.58$; MS $m / z: 273(\mathrm{M}+1)^{+}$. ESI-HRMS calcd for $\mathrm{C}_{14} \mathrm{H}_{14} \mathrm{ClN}_{4}$ $\left([\mathrm{M}+\mathrm{H}]^{+}\right)$273.0902, found 273.0909.

2-((2'-Bromo-[1,1'-biphenyl]-4-yl)methylene)hydrazine1-carboximidamide (3h): Light yellow solid, yield $78 \%$. m.p. $218 \sim 219{ }^{\circ} \mathrm{C} ;{ }^{1} \mathrm{H}$ NMR (DMSO- $\left.d_{6}, 400 \mathrm{MHz}\right) \delta: 5.79$ $\left(\mathrm{s}, 2 \mathrm{H}, \mathrm{NH}_{2}\right), 6.09\left(\mathrm{~s}, 2 \mathrm{H}, \mathrm{NH}_{2}\right), 7.29 \sim 7.77(\mathrm{~m}, 8 \mathrm{H}, \mathrm{ArH})$, $8.05(\mathrm{~s}, 1 \mathrm{H}, \mathrm{N}=\mathrm{CH}) ;{ }^{13} \mathrm{C} \mathrm{NMR}\left(\mathrm{DMSO}-d_{6}, 100 \mathrm{MHz}\right) \delta$ : $160.43,142.63,141.62,139.66,136.28,133.07,131.38$, 129.34, 129.26, 128.04, 125.93, 121.71; MS m/z: 317 (M+ $1)^{+}$. ESI-HRMS calcd for $\mathrm{C}_{14} \mathrm{H}_{14} \mathrm{BrN}_{4}\left([\mathrm{M}+\mathrm{H}]^{+}\right)$ 317.0396, found 317.0389.

2-((3'-Bromo-[1,1'-biphenyl]-4-yl)methylene)hydrazine1-carboximidamide (3i): Light yellow solid, yield $69 \%$. m.p. $206 \sim 208{ }^{\circ} \mathrm{C} ;{ }^{1} \mathrm{H}$ NMR (DMSO- $\left.d_{6}, 400 \mathrm{MHz}\right) \delta: 5.63$ $\left(\mathrm{s}, 2 \mathrm{H}, \mathrm{NH}_{2}\right), 6.03\left(\mathrm{~s}, 2 \mathrm{H}, \mathrm{NH}_{2}\right), 7.40 \sim 7.88(\mathrm{~m}, 8 \mathrm{H}, \mathrm{ArH})$, $8.03(\mathrm{~s}, 1 \mathrm{H}, \mathrm{N}=\mathrm{CH}) ;{ }^{13} \mathrm{C} \mathrm{NMR}$ (DMSO- $\left.d_{6}, 100 \mathrm{MHz}\right) \delta$ : $160.70,142.57,142.28,137.49,136.85,131.09,130.12$, $128.99,126.84,126.82,125.58,122.47$; MS m/z: $317(\mathrm{M}+$ $1)^{+}$. ESI-HRMS calcd for $\mathrm{C}_{14} \mathrm{H}_{14} \mathrm{BrN}_{4}\left([\mathrm{M}+\mathrm{H}]^{+}\right)$ 317.0396, found 317.0391.

2-((4'-Bromo-[1,1'-biphenyl]-4-yl) methylene)hydrazine1-carboximidamide (3j): Light yellow solid, yield $80 \%$. m.p. $219 \sim 221{ }^{\circ} \mathrm{C} ;{ }^{1} \mathrm{H}$ NMR (DMSO- $\left.d_{6}, 400 \mathrm{MHz}\right) \delta: 5.81$ $\left(\mathrm{s}, 2 \mathrm{H}, \mathrm{NH}_{2}\right), 6.11\left(\mathrm{~s}, 2 \mathrm{H}, \mathrm{NH}_{2}\right), 7.62 \sim 7.67(\mathrm{~m}, 6 \mathrm{H}, \mathrm{ArH})$, $7.78(\mathrm{~d}, J=8.3 \mathrm{~Hz}, 2 \mathrm{H}, \mathrm{ArH}), 8.04(\mathrm{~s}, 1 \mathrm{H}, \mathrm{N}=\mathrm{CH}) ;{ }^{13} \mathrm{C}$ NMR (DMSO- $\left.d_{6}, 100 \mathrm{MHz}\right) \delta: 160.36,142.69,138.94$, 137.91, 136.41, 131.80, 128.53, 126.89, 126.51, 120.80; MS $m / z: 317(\mathrm{M}+1)^{+}$. ESI-HRMS calcd for $\mathrm{C}_{14} \mathrm{H}_{14} \mathrm{BrN}_{4}$ $\left([\mathrm{M}+\mathrm{H}]^{+}\right)$317.0396, found 317.0398.

\subsection{Evaluation of anti-bacterial activity in vitro}

The anti-bacterial activity in vitro against $S$. aureus CMCC(B) 26003 and CMCC 25923, S. pyogenes CMCC 32067, E. faecalis CMCC 29212, B. subtilis CMCC 63501; E. coli $\mathrm{CMCC} 25922$ and CMCC 44568, P. aeruginosa CMCC 27853 and CMCC 10104, as well as two methicillin-resistant clinical isolates ( $S$. aureus ATCC 43300 and ATCC 33591) was evaluated using a two-fold serial dilution technique, and the final concentrations of compounds obtained were in the range of $0.5 \sim 128 \mu \mathrm{g} / \mathrm{mL}$. Test bacteria were grown to mid-log phase in Mueller-Hinton Broth (MHB) or Tryptone Soya Broth (TSB), and diluted 1000 -fold in the same medium. The bacteria of $10^{5} \mathrm{CFU} / \mathrm{mL}$ were inoculated into MHB or TSB and dispensed at 0.2 $\mathrm{mL} /$ well in a 96-well microtiter plate. As positive controls, 
gatifloxacin, moxifloxacin, norfloxacin, oxacillin, and penicillin were used. Test compounds were prepared in DMSO, the final concentration of which did not exceed $0.05 \%$. The MIC was defined as the concentration of a test compound that completely inhibited bacteria growth during $24 \mathrm{~h}$ incubation at $37{ }^{\circ} \mathrm{C}$. Bacteria growth was determined by measuring the absorption at $630 \mathrm{~nm}$ using a microtiter enzyme-linked immunosorbent assay (ELISA) reader. All experiments were carried out three times.

\subsection{Evaluation of cytotoxicity in vitro}

Human embryonic kidney 293 T cells (HEK 293 T cells) were used to test the anticancer activity of the new compounds. HEK 293T cells were grown in Dulbecco modified Eagle medium supplemented with fetal bovine serum $(10 \%)$, and antibiotics (penicillin-streptomycin mixture $(100 \mathrm{U} / \mathrm{mL}))$. Cells at $80 \%$ to $90 \%$ confluence were split by trypsin $(0.25 \%$ in PBS; $\mathrm{pH} 7.4)$, and the medium was changed at 24 -h intervals. The cells were cultured at $37{ }^{\circ} \mathrm{C}$ in a $5 \% \mathrm{CO}_{2}$ incubator. The cells were grown to three passages, and approximately $1 \times 10^{4}$ cells were seeded into each well of a 96-well plate and allowed to incubate to allow attachment of the cells to the substrate. After $24 \mathrm{~h}$, the medium was replaced with DMEM supplemented with 10\% FBS containing various concentrations $(0.3,1,3,10,30$, and $100 \mu \mathrm{mol} / \mathrm{L}$ ) of test compounds and incubated for $48 \mathrm{~h}$. For each concentration, three wells were set in parallel. Then, $20 \mu \mathrm{L}$ of CCK-8 solution was added to each well. After incubation for $3 \mathrm{~h}$, the optical density was measured at $450 \mathrm{~nm}$ using a microtiter ELISA reader. The $\mathrm{IC}_{50}$ values were defined as the concentrations inhibiting $50 \%$ of cell growth.

Supporting Information The ${ }^{1} \mathrm{H}$ NMR spectra of intermediates $\mathbf{2} \mathbf{a} \sim \mathbf{2 j}$, and ${ }^{1} \mathrm{H}$ NMR, ${ }^{13} \mathrm{C}$ NMR spectra and high resolution mass spectrum of target compounds $\mathbf{3 a} \sim \mathbf{3 j}$. The Supporting Information is available free of charge via the
Internet at http://sioc-journal.cn/.

\section{References}

[1] Bi, Y.; Liu, X. X.; Zhang, H. Y.; Yang, X.; Liu, Z. Y.; Lu, J.; Lewis, P. J.; Wang, C. Z.; Xu, J. Y.; Meng, Q. G.; Ma, C.; Yuan, C. S. Molecules 2017, 22, 590 .

[2] Carrel, M.; Perencevich, E. N.; David, M. Z. Emerging Infect. Dis. 2015, 21, 1973.

[3] Hvistendahl, M. Science 2012, 336, 795.

[4] Yezli, S.; Li, H. Int. J. Antimicrob. Agents 2012, 40, 389.

[5] Azeredo da Silveira, S.; Perez, A. Expert Rev. Anti-Infect. Ther 2015, 13, 531 .

[6] Kang, Y.; Zhao, M.; Zhang, J. Chin. J. Antibiot. 2017, 42, 169 (in Chinese).

(康悦，赵明，张菁，中国抗生素杂志, 2017, 42, 169.)

[7] Ward, R. S. Nat. Prod. Rep. 1999, 16, 75.

[8] Whiting, D. A. Nat. Prod. Rep. 2001, 18, 583.

[9] Chang, J. B.; Reiner, J.; Xie, J. X. Chem. Rev. 2005, 105, 4581.

[10] Chen, D. F.; Zhang S. X.; Lan, X. Bioorg. Med. Chem. 1997, 5, 715.

[11] Raffa, D.; Plescia, F.; Maggio, B.; Raimondi, M. V.; D'Anneo, A.; Lauricella, M.; Daidone, G. Eur. J. Med. Chem. 2017, 132, 262.

[12] Chen, Y.; Du, Y. J.; Suo, F. X. J. Henan Normal Univ. (Nat. Sci.) 2001, 29, 43 (in Chinese).

(陈勇, 杜燕军, 索福喜, 河南师范大学学报 (自然科学版), 2001, 29, 43.)

[13] Wei, Z. Y.; Chi, K. Q.; Yu, Z. K.; Liu, H. Y.; Sun, L. P.; Zheng, C. J.; Piao, H. R. Bioorg. Med. Chem. Lett. 2016, 26, 5920.

[14] Sidoryk, K.; Świtalska, M.; Rózga, P.; Wietrzyk, J.; Bujak, I.; Żerek, B.; Kaczmarek, Ł.; Cybulski, M. Med. Chem. Res. 2017, 26, 3354.

[15] Gao, Z. M.; Wang, T. T.; Li, S. Z.; Wan, H. Q.; Wang, G.; Wu, Y. B.; Deng, X. Q.; Song, M. X. Chin. J. Org. Chem. 2016, 36, 2484 (in Chinese).

(高智敏, 王田田, 李深圳, 万慧琪, 王刚, 吴银涁, 邓先清, 宋 明霞，有机化学, 2016, 36, 2484.)

[16] Li, Y. R.; Li, C.; Liu, J. C.; Guo, M.; Zhang, T. Y.; Sun, L. P.; Zheng, C. J.; Piao, H. R. Bioorg. Med. Chem. Lett. 2015, 25, 5052.

[17] Xu, H.; Wang, Y. Y. Bioorg. Med. Chem. Lett. 2010, 20, 7274

[18] Mourer, M.; Dibama, H. M.; Fontanay, S.; Grare, M.; Duval, R. E.; Finance, C.; Vains, J. B. R. Bioorg. Med. Chem. 2009, 17, 5496. 